\title{
Tick-borne encephalitis in north-east Italy: a 14-year retrospective study, January 2000 to December 2013
}

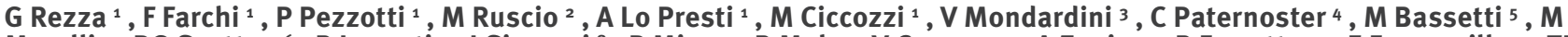

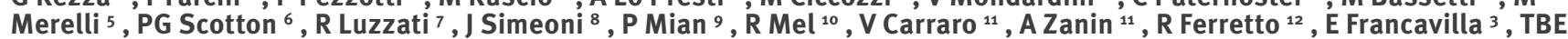 \\ Virology Group ${ }^{13}$ \\ 1. Department of Infectious, Parasitic and Immunomediated Diseases, National Institute of Health, Rome, Italy \\ 2. Unit of Laboratory Medicine, Ospedale Sant' Antonio ASS 4 'Medio Friuli', S. Daniele del Friuli, Udine, Italy \\ 3. Division of Infectious Diseases, ULSS 1 Belluno Hospital, Belluno, Italy \\ 4. Division of Infectious Diseases, Santa Chiara Hospital, Trento, Italy \\ 5. Infectious Diseases Division, Santa Maria della Misericordia Hospital, Udine, Italy \\ 6. Division of Infectious Diseases, Santa Maria di Cà Foncello Hospital, Treviso, Italy \\ 7. Infectious Disease Unit, University Hospital of Trieste, Trieste, Italy \\ 8. Department of Health of the Autonomous Province of South Tyrol, Bozen, Italy \\ 9. Division of Infectious Diseases, Bolzano Hospital, Treviso, Italy \\ 10. Department of Public Health, Belluno, Italy \\ 11. Department of Public Health, Trento, Italy \\ 12. Division of Infectious Diseases, Alto Vicentino Hospital, Santorso (VI), Italy \\ 13. The members of the group are listed at the end of the article
}

Correspondence: Giovanni Rezza (giovanni.rezza@iss.it)

Rezza G, Farchi F, Pezzotti P, Ruscio M, Lo Presti A, Ciccozzi M, Mondardini V, Paternoster C, Bassetti M, Merelli M, Scotton PG, Luzzati R, Simeoni J, Mian P, Mel R, Carraro V, Zanin A, Ferretto R, Francavilla E. Tick-borne encephalitis in north-east Italy: a 14-year retrospective study, January 2000 to December 2013 . Euro Surveill. 2015;20(40):pii=30034. DOI: http://dx.doi.org/10.2807/1560-7917.ES.2015.20.40.30034

Italy is considered at low incidence of tick-borne encephalitis (TBE), and the occurrence of human cases of TBE appears to be geographically restricted to the north east of the country. However, most information to date derives from case series, with no systematic data collection. To estimate incidence rates (IR) and spatial distribution of TBE cases, we conducted a retrospective study in north-eastern Italy. Data were collected through the infectious disease units and public health districts of three regions (Friuli Venezia Giulia, Trentino Alto Adige and Veneto) between 2000 and 2013. Overall, 367 cases were identified (IR: $0.38 / 100,000)$. The cases' median age was 56 years and 257 (70\%) were male. Central nervous system involvement was reported in 307 cases (84\%). Annual fluctuations in case numbers occurred, with peaks in 2006 and in 2013, when 44 and 42 cases were respectively observed. A strong seasonality effect was noted, with the highest number of cases in July. In terms of geographical location, three main endemic foci with high TBE IR (>10/100,000) were identified in three provinces, namely Belluno (Veneto region), Udine (Friuli Venezia Giulia) and Trento (Trentino AltoAdige). When investigating the whole study area in terms of altitude, the IR between 400 and $600 \mathrm{~m}$ was greater $(2.41 / 100,000)$ than at other altitudes ( $p$ s 0.01). In conclusion, the incidence of TBE in Italy is relatively low, even considering only the three known affected regions. However, three endemic foci at high risk were identified. In these areas, where the risk of
TBEV infection is likely high, more active offer of TBE vaccination could be considered.

\section{Introduction}

Tick-borne encephalitis (TBE) is considered a disease of the central nervous system (CNS) caused by infection with the TBE virus (TBEV), a flavivirus discovered in 1937, during an expedition in Far-East Russia [1].

There are three TBEV subtypes: the European, the Siberian, and the Far Eastern subtype. The main hosts and reservoirs of TBEV are small rodent species, whereas ticks (Ixodes persulcatus and I. ricinus) act as the vector [2]. The principal vector of the European TBEV subtype is /. ricinus.

The clinical manifestations of TBE usually start with a febrile illness, but ca 20 to $30 \%$ of the patients subsequently develop CNS disease, such as meningitis or encephalitis [2-4]. Thus, the neurological manifestation defining the syndromic picture of TBE underrepresent the overall clinical burden of disease related to TBEV infection.

The natural habitat of TBEV is represented by the forests of Europe and Asia. In the European continent, TBEV is distributed in an endemic pattern of so-called natural foci over central and north-eastern Europe, with the highest incidence $(>10$ cases/100,000) in Baltic countries, the Czech Republic, Russia, and Slovenia [5]. In 
TABLE 1

Descriptive characteristics of persons diagnosed with tick-borne encephalitis in 'Triveneto', north-eastern Italy, $2000-2013(n=367)$

\begin{tabular}{|c|c|c|}
\hline \multicolumn{2}{|c|}{ Characteristics } & $\mathrm{N}(\%)^{\mathrm{a}}$ \\
\hline \multirow{2}{*}{ Nationality } & Italians & $355(96.7)$ \\
\hline & Others & $12(3.3)$ \\
\hline \multirow{2}{*}{ Area of residence } & Living in north-eastern Italy & $364(99.2)$ \\
\hline & Living in other areas & $3(0.8)$ \\
\hline \multirow{2}{*}{ Sex } & Female & $110(30.0)$ \\
\hline & Male & $257(70.0)$ \\
\hline Age & Median (IQR) & $56(42-67)$ \\
\hline \multirow{12}{*}{$\begin{array}{l}\text { Month of onset } \\
\text { symptoms }\end{array}$} & January & $2(0.5)$ \\
\hline & February & $1(0.3)$ \\
\hline & March & $3(0.8)$ \\
\hline & April & $25(6.8)$ \\
\hline & May & $50(13.6)$ \\
\hline & June & $64(17.4)$ \\
\hline & July & $79(21.5)$ \\
\hline & August & $44(12.0)$ \\
\hline & September & $32(8.7)$ \\
\hline & October & $53(14.4)$ \\
\hline & November & $11(3.0)$ \\
\hline & December & $3(0.8)$ \\
\hline \multirow{5}{*}{ Clinical syndrome } & Encephalitis & $175(47.7)$ \\
\hline & Meningoencephalitis & $94(25.6)$ \\
\hline & Febrile illness only & $60(16.4)$ \\
\hline & Aseptic meningitis & $25(6.8)$ \\
\hline & Meningoencephalomyelitis & $13(3.5)$ \\
\hline Vaccinated & - & $3(0.8)$ \\
\hline Sequelae & - & $60(16.3)$ \\
\hline Deaths & - & $2(0.5)$ \\
\hline
\end{tabular}

IQR: interquartile range.

a Unless otherwise specified.

Austria, which is the only country with a significantly decreasing trend of TBE cases, due to mass vaccination programmes, the incidence in the unvaccinated population is ca five per $100,000[5,6]$.

Italy is considered a country with geographically restricted, low incidence of TBE [6]. The disease is not notifiable in the country but individuals with TBE can be reported at the regional level, where they are classified as 'viral meningitis and encephalitis' (notifiable diseases, class 2). Although the extent of affected areas in Italy is not well defined, the regions of the north east appear to be those where cases have been documented, as suggested by case series of TBE published in the last decade [7-10]. However, to the best of our knowledge, systematic information on the occurrence of persons with TBE is lacking even in these affected areas of Italy, and both the incidence rates (IR) and the geographical distribution of persons with the disease have been mostly derived from international literature or reports of aggregated cases [6].
In the European Union (EU), TBE was added to the list of notifiable diseases in 2012 with a harmonised case definition focused on neuroinvasive illness with laboratory confirmation [11], highlighting the need of detailed epidemiological data.

To fill the gap in knowledge about TBE distribution in Italy, we conducted a retrospective study in three affected regions (Friuli Venezia Giulia, Trentino Alto Adige and Veneto) in the north-east of the country. The study was aimed at estimating the incidence of TBE (including febrile illness and/or CNS disease) in these regions from 2000 to 2013 . We also sought to identify at risk areas within the affected regions, and to describe demographic parameters of TBE cases. Some environmental factors were also investigated.

\section{Methods}

\section{Study area}

The study was conducted in three north-eastern Italian regions: Friuli Venezia Giulia, Trentino-Alto Adige (i.e. the autonomous provinces of Trento and Bolzano), and Veneto. Together, these regions, which cover $39,728 \mathrm{~km}^{2}$, and at the beginning of 2013 comprised around 7.1 million inhabitants, are commonly referred to as 'Triveneto'. The northern and eastern parts of the 'Triveneto' area border with Austria and Slovenia respectively and have a mountainous landscape (the Alps), while the southern part comprises the eastern part of the Po valley. The area is administratively divided in around 1,130 municipalities and their altitude shows a strong gradient increasing from south to north. The study area was identified as that of major interest for TBE through a literature review (i.e. PubMed search using 'tick-borne encephalitis' or 'TBE' or 'TBEV' and 'Italy' as keywords, in addition to revision of grey literature, such as national and EU reports), and consultations with experts in the field. Entomological data (i.e. the distribution of TBEV infected ticks) was also revised using a similar approach (PubMed searches, examination of grey literature as well as reliance on expert opinion). On the basis of this information, the expected area of interest for TBE was only a fraction of the three regions; however, we included contiguous areas in the study, to exclude the occurrence of autochthonous cases, and to identify possible cases in individuals travelling to, and/or spending their vacations in the affected areas. Public health authorities and clinicians of other regions of northern Italy (i.e. those bordering regions or countries reporting TBE cases) were also contacted during the study to exclude the presence of known persons affected by TBE.

\section{Data collection}

To gather information on cases of TBE diagnosed between January 2000 and December 2013, all the infectious diseases units and public health districts in the study area were contacted. Study visits were planned to support clinicians in standardised data collection. Clinical registers were accurately checked; 


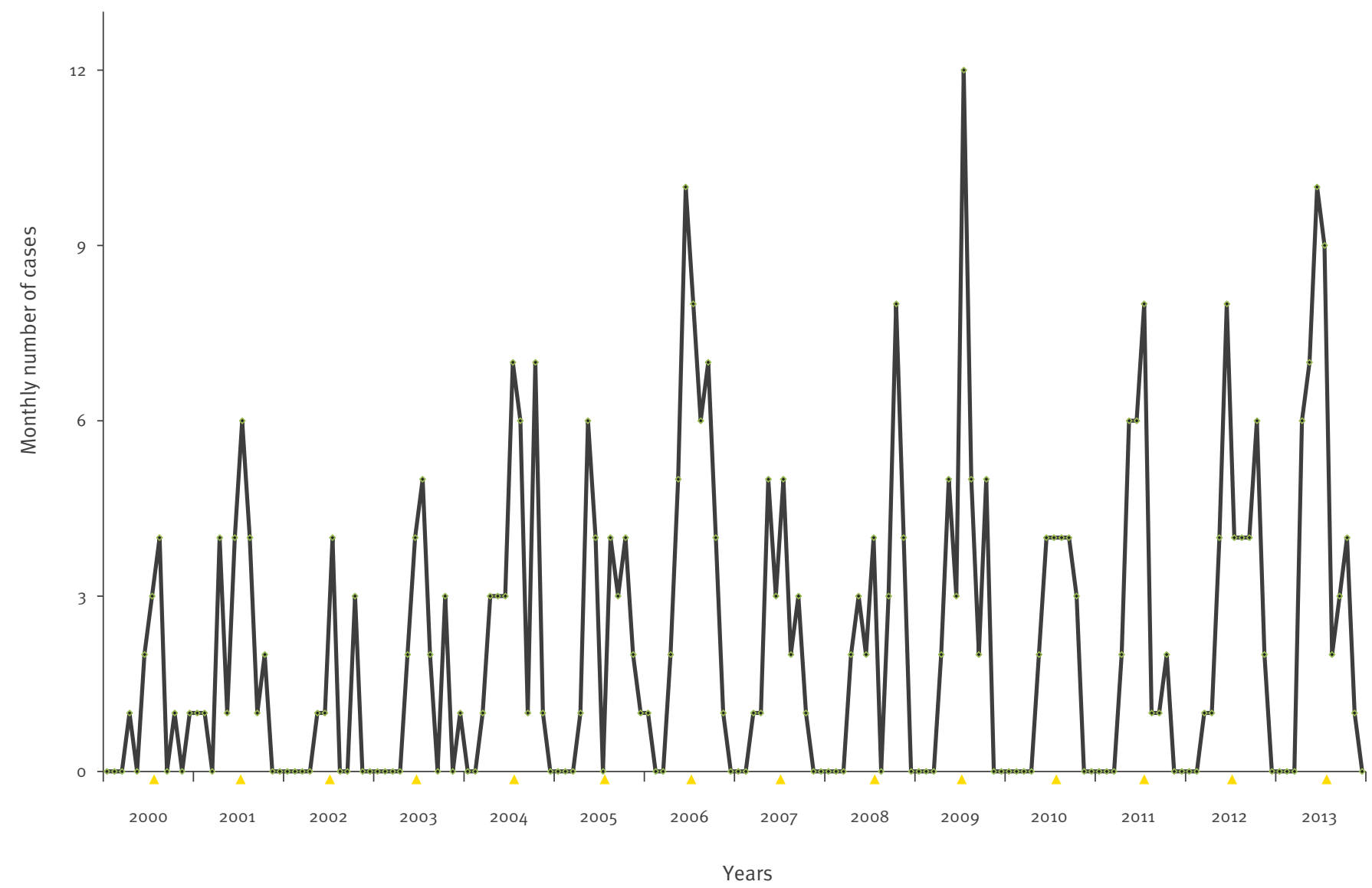

The yellow marks (triangles) indicate the month of July of each year, as during this month a higher number of cases was frequently observed.

when needed, laboratory registries were revised by trained staff. Collection of information on TBE affected persons was guided by a questionnaire relating to demographic data (age, sex, place of residence, and nationality), clinical information (i.e. febrile illness, meningitis, encephalitis, meningoencephalitis, other neurological symptoms), and presumed place of exposure to tick bite. When available from the clinical register, data on environmental factors or behaviour, which could potentially have led to tick exposure were also gathered.

\section{Case definition}

To the purpose of this study, we adopted a case definition based on clinical and epidemiological criteria, similar to some of those used in other EU Member States [11].

A case of TBE was defined by the presence of a febrile illness, with/without encephalitis, meningitis, meningoencephalitis or meningoencephalomyelitis, and laboratory criteria (i.e. specific IgM and IgG response to TBEV, or seroconversion, or fourfold increase in IgG, or detection of TBEV nucleic acid by polymerase chain reaction).

\section{Statistical analysis}

Descriptive techniques, such as frequency distributions, percentages, medians, and interquartile ranges, were used to summarise the data. As measure of TBE occurrence, IRs were then calculated as the number of cases per 100,000 individuals of population at risk. This population was considered that reported by the Italian National Bureau of Census (www.demo.istat.it) as individuals living at the beginning of each year in the study regions. IRs were also stratified by province and municipality of residence. The temporal trend was evaluated using Poisson regression; incidence rate ratios (IRR) per one calendar year were then calculated. IRRs by Poisson regression were also calculated to evaluate the association with the altitude of the municipality. IR per municipality of residence were graphically represented as geographical maps.

\section{Results}

During the period from 2000 to 2013,367 cases (0.38/100,000 inhabitants) were diagnosed in the study area. Table 1 shows the main characteristics of the cases. Almost all of them $(n=355)$ were Italian nationals and a majority of cases $(n=364)$ was also residing in the study area; cases were mainly male $(n=257$; $70 \%)$, and around $70 \%(n=255)$ were between 30 and 
Annual incidence rates (per 100,000 inhabitants) of tick-borne encephalitis cases by municipalities of residence in northeastern Italy, 2000-2013

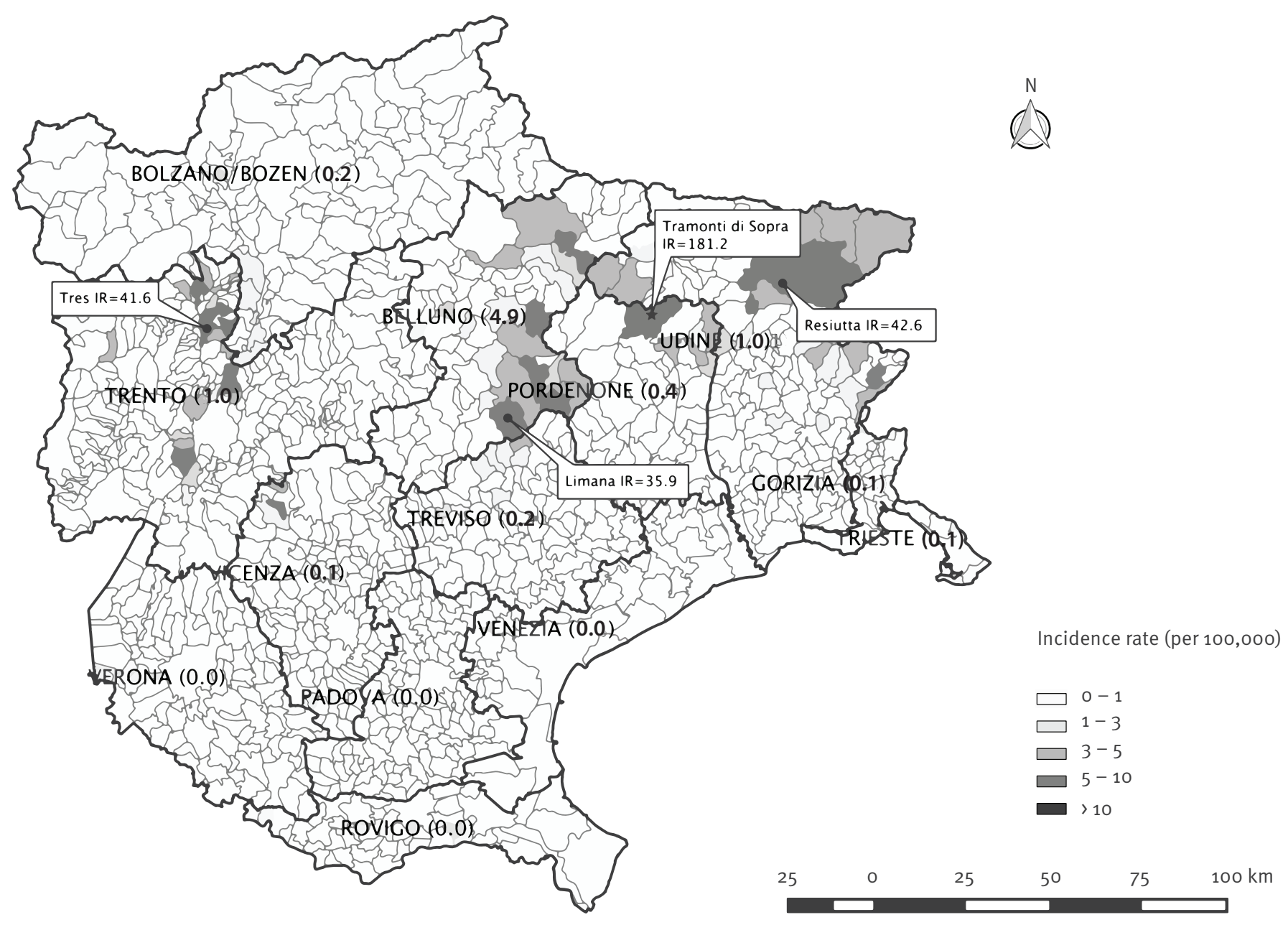

IR: incidence rate.

Numbers in parentheses are the IRs per province of residence. For some municipalities with high IRs, the names of the municipalities and the corresponding IR values are shown.

70 years-old. Encephalitis was the most common clinical presentation ( $n=175$ cases), while febrile syndrome without CNS involvement ( $n=60$ cases) accounted for less than a sixth of the cases. Symptom onset occurred mainly between April and October ( $n=347$ cases; $94.5 \%)$. Almost all cases $(n=364)$ were not vaccinated and whether full vaccination was achieved among those who were is not known. Sequelae were reported in $60(16.3 \%)$ individuals. Of them, 23 developed tiredness, 19 paresis, 11 tremor, seven headache, six memory and/or concentration disturbances, three pain in the extremities; insomnia, anxiety, or nausea were reported by two patients, respectively, whereas hearing disturbance, dizziness, or electroencephalography anomalies only by one (data not shown in table). Two cases died (case fatality rate: $0.5 \%$ ).

Modality of tick bite exposure was available for 156 of the 367 cases. Recreational activities were mentioned by 111 cases; of them, some specified trekking $(n=25)$, mushrooming $(n=16)$, whereas only five hunting. Living in a high risk area was reported by 32 individuals (for 6 of them it was a summer house). Nine cases were agricultural workers. Finally, four cases reported probable tick-bites while abroad ( 2 in Austria and 2 in Slovenia, respectively).

Figure 1 shows the distribution of the number of cases per month, during the study period. A strong seasonality could be observed, with the number of cases usually peaking in July, and in large part occurring between April and October (see also Table 1). Considering the annual IR, a significant temporal increase was observed during the study period, with annual IR increasing from 0.18 in the year 2000 to 0.59 per 100,000 in 2013 $(I R R=1.05$ per one calendar year increase; $95 \%$ confidence interval $(\mathrm{Cl}): 1.02-1.08, \mathrm{p}<0.01$ ) (data not shown in Figure 1); however, the overall increase was mainly 
TABLE 2

Incidence rates (IR) and incidence rate ratios (IRR) of tick-borne encephalitis (TBE) in 'Triveneto' according to the altitude of the municipalities of residence, north-eastern Italy, 2000-2013

\begin{tabular}{|c|c|c|c|c|c|c|}
\hline $\begin{array}{l}\text { Municipality altitude } \\
\text { (metres) }\end{array}$ & $\begin{array}{c}\text { Number of } \\
\text { municipalities }\end{array}$ & $\begin{array}{c}\text { Municipalities with at } \\
\text { least one case } \\
\text { N (\%) }\end{array}$ & $\begin{array}{l}\text { Total TBE } \\
\text { cases }\end{array}$ & $\begin{array}{l}\text { IR (per } \\
100,000)\end{array}$ & $\begin{array}{c}\text { IRR } \\
(95 \% \mathrm{Cl})\end{array}$ & P-value ${ }^{a}$ \\
\hline $0-200$ & 589 & $44(7 \cdot 5)$ & 78 & 0.10 & $1.00(-)$ & $<0.01$ \\
\hline $200-400$ & 146 & $40(27.4)$ & 162 & 1.54 & $15.16(11.57-19.86)$ & $<0.01$ \\
\hline $400-600$ & 101 & $22(21.8)$ & 70 & 2.41 & $23.81(17.24-32.87)$ & - \\
\hline $600-800$ & 103 & $15(14.6)$ & 28 & 1.27 & $12.52(8.13-19.27)$ & $<0.01$ \\
\hline$>800$ & 196 & $17(8.7)$ & 26 & 0.55 & $5.42(3.48-8.45)$ & $<0.01$ \\
\hline
\end{tabular}

a P-values refer to comparisons between IR in municipalities with a mean altitude between 400 and $600 \mathrm{~m}$ with that of the municipalities with another mean altitude.

due to the change in incidence that occurred between 2003 (IR: 0.25/100,000) and 2004 (IR: 0.48/100,000). In fact, after excluding the years before 2004, the estimated IRR was 1.01 (95\%Cl: 0.97-1.05, $\mathrm{p}=0.64$ ). Similar results were obtained when excluding from the analysis cases with only febrile illness (data not shown).

Figure 2 shows the map of the mean annual IR by municipality of residence. Noteworthy, the municipality with the highest IR in the entire area was Tramonti di Sopra (181.2/100,000) in the province of Pordenone. At the province level, the map clearly identifies three provinces with the highest IR: Belluno province (Veneto region), of which the municipality of Limana presented the highest IR (35.9/100,000), Udine province (Friuli Venezia Giulia region), with the municipality of Resiutta having the highest IR (42.6), and the Trento province (Trentino Alto-Adige region), with the highest IR observed in the municipality of Tres (41.6). The highest number of cases was also reported in these three provinces; in particular, the most affected municipalities were Belluno ( $n=49$ cases), Limana $(n=24)$, and Ponte nelle Alpi $(n=17)$, all located in the province of Belluno (data not shown).

Table 2 summarises the number of cases, the estimated $I R$, and the IRR, after stratifying the municipalities of residence by altitude. Overall, there was a significant association in terms of altitude, with the lowest IR value in municipalities at an altitude $\leq 200 \mathrm{~m}$ and the highest in municipalities between 400 and $600 \mathrm{~m}$. For altitudes above $600 \mathrm{~m}$, IR decreased with further municipality altitude increase; however, the IR for municipalities with a mean altitude $>800 \mathrm{~m}$ remained five times significantly higher than for municipalities with a mean altitude of $\leq 200 \mathrm{~m}$.

\section{Discussion}

Italy is considered a country at low risk for TBE, which appears to be restricted to a relatively small fraction of its territory. However to date, accurate information on the occurrence of TBE and/or TBEV infection in Italy is not available, and the major source of data has originated from case reports or case series [6-10], all from the north-eastern regions, and especially from areas bordering high incidence countries such as Austria and Slovenia. Thus, our data allow, for the first time, to accurately calculate incidence rates and to describe the geographical distribution of TBE cases in the affected Italian areas.

The total number and IR of cases found in our study appear to be much higher than that reported in the past, presumably from the same geographical area. For example, according to an article summarising data from different European countries, only 102 cases were observed in Italy in the period from 1975 to 2001; of these, 14 occurred between 1975 and 1991, and 88 from 1992 to 2001 [12]. However, a direct comparison with these data is not possible, since their source and the study area were not well defined.

We found an IR of ca 0.4 per 100,000 in the overall study area. However, at the level of the most affected provinces of Trento, Belluno, and Udine, the IR ranged between 1 and 5 per 100,000 which appears to be lower than that reported in neighbouring countries such as Austria (before mass vaccination) and Slovenia [6]. In particular, incidence rates of 17.9 per 100,000 (ranging from 1.2 to $42.8 / 100,000$ over the last four decades) and eight per 100,000 have been reported respectively in the unvaccinated population of Carinthia and Tirol, two Austrian regions bordering ‘Triveneto' [13].

The annual number of cases observed in 'Triveneto' tended to vary, showing two peaks respectively in 2006 and 2013, whereas the lowest number of cases was registered in 2002 . Yearly trend fluctuations were observed also in other countries such as Switzerland, where a study from 2005 to 2011 found the lowest and the highest number of cases in 2002 and 2006, respectively [14].

Most cases occurred between April and October, with peaks in June and July. This is rather consistent with the seasonality of tick activity, with slight differences compared with central Europe, where seasonal peaks 
of feeding activity of ticks tend to occur between May and June, and between September and October [1].

The demographic characteristics of our patients, showing male predominance and rather advanced median age, were similar to those observed in neighbouring countries such as Switzerland, where TBE incidence peaked in 60-69 year-olds, and male predominance was observed in all age groups [14].

Most cases were concentrated in three main foci, one in the autonomous province of Trento, one in the pre-alpine Belluno province of Veneto, and one at the extreme north-east of Friuli Venezia Giulia. In this respect, it should be underlined that TBEV epidemiology is closely associated with ticks' ecology, and that ticks inhabit specific foci in the forests with enhanced moist vegetation and wild animals supplying blood meals for them [1]. The special combination of climatic conditions, including temperature, moisture, and vegetation, occurring only within specific geographical zones, provides an heterogeneous tick distribution, generating as many as 20,000 or even 30,000 estimated natural foci of TBEV infections across the northern hemisphere [1].

The heterogeneous geographical distribution of human cases in our study appears to be consistent with that of competent vectors, as suggested by entomological studies conducted in the same area. To this regard, TBEV was detected in $2.1 \%$ of 193 ticks collected in 14 sites of north-eastern Italy between April and June in the years 2006 to 2008 [15]. Further studies showed that the density distribution of competent ticks and the prevalence of TBEV infection is not homogeneously distributed within the affected regions. In fact, $0.21 \%$ of 2,361 of I. ricinus ticks collected in the alpine area of the extreme north-eastern Italy (Friuli Venezia Giulia) between 2005 and 2006 were positive for TBEV, but all of them were found in three sites where the highest tick density was found [16].

Recreational activities were found to be the most common possible exposure to tick bite in our study; however, almost all the cases were reported among residents. This might be due to the fact that, though the Alps represent a touristic area, tourists tend to spend their vacations at higher altitudes than those found to be at highest risk. To this regard, a case-control study conducted in Poland found that, in endemic areas, the highest TBE risk was associated with spending at least 10 hours a week in mixed forests and harvesting forest foods, being unemployed, or employed as a forester [17].

TBEV infection may be prevented not only adopting behavioural precautions, but also by using effective vaccines. In our study, only three individuals reported having been vaccinated against TBE; however, whether they had completed the full vaccination schedule is unknown. In Austria, data collected in the period between 1972 and 2011 showed that mass vaccination reduced incidence to ca $16 \%$ of that of the prevaccination era: average incidence declined from 5.7 per 100,000 in the first ten years to 0.9 per 100,000 in the last decade of the study, while TBE vaccination, which started in 1972 , reached $88 \%$ in 2005 [18]. TBE vaccination is recommended to high risk population groups (foresters, scouts, persons with hobbies or leisure activities potentially leading to tick exposure) in Veneto and in Trentino Alto Adige. In Friuli Venezia Giulia, vacccination has been offered free of charge to all the population of the region since 2013; however, whether vaccination had any impact on the disease burden in north-eastern Italy remains undefined.

Before drawing conclusions, some limits and possible biases of our study should be mentioned. Firstly, our case-definition included also febrile illness without CNS involvement. This may supply a more comprehensive figure of TBE cases and their distribution, but it makes the data less reliable since a large proportion of febrile cases could have not been diagnosed; moreover, the proportion of undetected cases may vary unpredictably in time and space. Secondly, we cannot rule out completely the presence of TBE foci in other Italian regions. To this purpose, it should be reminded that in the 1970s, a few TBE cases and infected ticks were found in Tuscany $[19,20]$. However, recent studies on the aetiology of meningoencephalitis have not confirmed these findings (data not shown). Moreover, studies of forestry rangers showed a seropositivity of $0.6 \%$ for TBE in the north-eastern region of Friuli Venezia Giulia [21], while none of those investigated in Tuscany or Lazio (both regions are located in central Italy) had TBE antibodies $[22,23]$. Finally, we cannot exclude completely the occurrence of a few cases in other regions of northern Italy, such as Lombardy, bordering Switzerland, another country at relatively high incidence of TBE. However, as reported above, active surveillance failed to identify human cases of TBE. These findings highly suggest that TBEV infection is restricted to the north-eastern area of the country, and it does not seem for the moment to expand its range to other Italian areas. The absence of cases away from the Alps area, even in the surveyed provinces of the three affected regions of north-eastern Italy, strengthens this argument.

In conclusion, seasonal occurrence of human cases of TBE in three regions of north-eastern Italy is confirmed. The incidence is relatively low, when compared with other countries in central and northern Europe. However, three endemic foci where risk of TBEV infection is likely high, were identified. Moreover the highest IRs were between 400 and $600 \mathrm{~m}$ of altitude. Most cases were detected among residents performing recreational activities. More active offer of anti-TBE vaccine to people living in high risk areas might be considered. 


\section{TBE Virology Group}

TBE Virology Group: Maria Grazia Ciufolini, Maria Elena Remoli, Cristiano Fiorentini, Antonella Marchi, Claudia Fortuna: Department of Infectious, Parasitic and Immunomediated Diseases, National Institute of Health, Rome, Italy. Pierlanfranco D'agaro: University of Trieste, Trieste, Italy. Cristina Pedrotti, Danila Bassetti: Trento Hospital, Treviso, Italy. Elisabetta Pagani: Laboratory of Microbiology and Virology, Bolzano Health District, Bolzano, Italy.

\section{Acknowledgements}

The study has been funded in part by a grant of the CCMMinistry of Health (ArboNet Project). We thank Dr. Luca Busani for his help with the realisation of Figure 2.

\section{Conflict of interest}

None declared.

\section{Authors' contribution}

GR had the original idea and wrote the first draft; FF, MC, and ALP supervised data collection, and FF also organised the study and managed the database; PP performed statistical analysis and revised the manuscript; all the other authors contributed to patient diagnosis and data collection at the local level.

\section{References}

1. GritsunTS, LashkevichVA, GouldEA. Tick-borne encephalitis. Antiviral Res. 2003;57(1-2):129-46. DOI: 10.1016/S01663542(02)00206-1 PMID: 12615309

2. DumpisU, CrookD, Oksij. Tick-borne encephalitis.Clin Infect Dis. 1999;28(4):882-90. DOI: 10.1086/515195 PMID: 10825054

3. HaglundM, GüntherG. Tick-borne encephalitis--pathogenesis, clinical course and long-term follow-up.Vaccine. 2003;21(Suppl 1):S11-8. DOI: 10.1016/S0264-410X(02)00811-3 PMID: 12628810

4. GustafsonR, SvenungssonB, ForsgrenM, GardulfA, GranströmM. Two-year survey of the incidence of Lyme borreliosis and tick-borne encephalitis in a high-risk population in Sweden.Eur J Clin Microbiol Infect Dis. 1992;11(10):894-900. DOI: 10.1007/BF01962369 PMID: 1486884

5. Donoso MantkeO, EscadafalC, NiedrigM, PfefferM, Working Group For Tick-Borne Encephalitis VirusC. Tickborne encephalitis in Europe, 2007 to 2009. Euro Surveill. 2011;16(39):19976.PMID: 21968423

6. European Centre for Disease Prevention and Control (ECDC). Epidemiological situation of tick-borne encephalitis in the European Union and European Free Trade Association countries. Stockholm: ECDC; 2012.

7. Zambito MarsalaS, PistacchiM, GioulisM, MelR, MarchiniC, FrancavillaE. Neurological complications of tick borne encephalitis: the experience of 89 patients studied and literature review.Neurol Sci. 2014;35(1):15-21. DOI: 10.1007/ S10072-013-1565-8 PMID: 24170165

8. CruciattiB, BeltrameA, RuscioM, VialeP, GigliGL. Neurological manifestation of tick-borne encephalitis in North-Eastern Italy. Neurol Sci. 2006;27(2):122-4. DOI: 10.1007/s10072-006-0612-0 PMID: 16816910

9. BeltrameA, ScudellerL, CristiniF, RoratoG, VialeP, CruciattiB, et al. Tick-borne encephalitis in Friuli Venezia Giulia, northeastern Italy. Infection. 2005;33(3):158-9. DOI: 10.1007/ S15010-005-4109-1 PMID: 15940419

10. BeltrameA, RuscioM, CruciattiB, LonderoA, Di PiazzaV, CopettiR, et al. Tickborne encephalitis virus, northeastern Italy. Emerg Infect Dis. 2006;12(10):1617-9. DOI: 10.3201/ eid1210.060395 PMID: 17176593

11. Amato-GauciA, ZellerH. Tick-borne encephalitis joins the diseases under surveillance in the European Union.Euro Surveill. 2012;17(42):20299.PMID: 23098821
12. SüssJ. Epidemiology and ecology of TBE relevant to the production of effective vaccines.Vaccine. 2003:21(Suppl 1):S1935. DOI: 10.1016/So264-410X(02)00812-5 PMID: 12628811

13. HeinzFX, StiasnyK, HolzmannH, KundiM, SixlW, WenkM, et al. Emergence of tick-borne encephalitis in new endemic areas in Austria: 42 years of surveillance. Euro Surveill. 2015;20(13):21077. DOI: 10.2807/1560-7917.ES2015.20.13.21077 PMID: 25860391

14. SchulerM, ZimmermannH, AltpeterE, HeiningerU. Epidemiology of tick-borne encephalitis in Switzerland, 2005 to 2011. Euro Surveill. 2014;19(13):20756. DOI: 10.2807/1560-7917. ES2014.19.13.20756 PMID: 24721541

15. CapelliG, RavagnanS, MontarsiF, CiocchettaS, CazzinS, PorcellatoE, et al. Occurrence and identification of risk areas of Ixodes ricinus-borne pathogens: a cost-effectiveness analysis in north-eastern Italy. Parasit Vectors. 2012;5(1):61. DOI: 10.1186/1756-3305-5-61 PMID: 22452970

16. D’AgaroP, MartinelliE, BurgnichP, NazziF, Del FabbroS, lobA, et al. Prevalence of tick-borne encephalitis virus in Ixodes ricinus from a novel endemic area of North Eastern Italy. J Med Virol. 2009;81(2):309-16. DOI: 10.1002/jmv.21389 PMID: 19107965

17. StefanoffP, RosinskaM, SamuelsS, WhiteDJ, MorseDL, RandolphSE. A national case-control study identifies human socio-economic status and activities as risk factors for tickborne encephalitis in Poland.PLoS ONE. 2012;7(9):e45511. DOI: 10.1371/journal.pone.0045511 PMID: 23029063

18. HeinzFX, StiasnyK, HolzmannH, Grgic-VitekM, KrizB, EsslA, et al. Vaccination and tick-borne encephalitis, central Europe. Emerg Infect Dis. 2013;19(1):69-76. DOI: 10.3201/ eid1901.120458 PMID: 23259984

19. Amaduccil, ArnetoliG, InzitariD, BalducciM, VeraniP, LopesMC. Tick-borne encephalitis (TBE) in Italy: report of the first clinical case.Riv Patol Nerv Ment. 1976;97(2):77-80.PMID: 1028140

20. PaciP, LeonciniF, MazzottaF, MiloD, AmaducciL, FratiglioniL, et al. Meningoencefaliti da zecche (TBE) in Italia [Tickborne meningoencephalitis (TBE) in Italy]. Ann Sclavo. 1980;22(3):404-16.PMID: 7247491

21. CincoM, BarboneF, Grazia CiufoliniM, MascioliM, Anguero RosenfeldM, StefanelP, et al. Seroprevalence of tick-borne infections in forestry rangers from northeastern Italy. Clin Microbiol Infect. 2004;10(12):1056-61. DOI: 10.1111/j.14690691.2004.01026.x PMID: 15606631

22. TomaoP, CiceroniL, D’OvidioMC, De RosaM, VoneschN, lavicolis, et al. Prevalence and incidence of antibodies to Borrelia burgdorferi and to tick-borne encephalitis virus in agricultural and forestry workers from Tuscany, Italy. Eur J Clin Microbiol Infect Dis. 2005;24(7):457-63. DOI: 10.1007/s10096005-1348-o PMID: 15948001

23. Di RenziS, MartiniA, BinazziA, MarinaccioA, VoneschN, D'AmicoW, et al. Risk of acquiring tick-borne infections in forestry workers from Lazio, Italy. Eur J Clin Microbiol Infect Dis. 2010;29(12):1579-81. DOI: 10.1007/S10096-010-1028-6 PMID: 20714765 